\title{
Géraldine FARGES, Les Mondes enseignants. Identités et clivages
}

\section{Ghislain Leroy}

\section{(2) OpenEdition}

1 Journals

Édition électronique

URL : https://journals.openedition.org/ress/3968

DOI : $10.4000 /$ ress.3968

ISSN : 1663-4446

Éditeur

Librairie Droz

\section{Édition imprimée}

Date de publication : 14 décembre 2018

Pagination : 299-301

ISSN : 0048-8046

\section{Référence électronique}

Ghislain Leroy, "Géraldine FARGES, Les Mondes enseignants. Identités et clivages », Revue européenne des sciences sociales [En ligne], 56-2 | 2018, mis en ligne le 14 décembre 2018, consulté le 06 janvier 2022. URL : http://journals.openedition.org/ress/3968; DOI : https://doi.org/10.4000/ress.3968

Ce document a été généré automatiquement le 6 janvier 2022.

(c) Librairie Droz 


\title{
Géraldine FARGES, Les Mondes enseignants. Identités et clivages
}

\author{
Ghislain Leroy
}

\section{RÉFÉRENCE}

Géraldine FARGES, 2017, Les Mondes enseignants. Identités et clivages, Paris, PUF, 240 p.

1 La problématique centrale de cet ouvrage de Géraldine Farges sur les "mondes enseignants » est résumée dans son sous-titre : «Identités et clivages ». Elle peut être ainsi reformulée: le monde enseignant est-il un ou multiple? Il s'agit tout particulièrement d'étudier les identités et différences entre le premier et le second degré, l'enseignement supérieur ne faisant pas partie des objets ici traités. De fait, de nombreuses études unifient le groupe social des enseignants, comme celles sur le « vote enseignant " par exemple (p.17). De même, des expressions répandues comme «les profs » ou encore « les enseignants » tendent à rassembler dans un groupe commun les enseignants des premier et second degrés. Enfin, d'un point de vue institutionnel, des termes tels que "communauté éducative» semblent sous-tendus par une même logique unificatrice. Pour autant, ce livre tend bien plutôt à montrer que le rapprochement entre les deux degrés relève surtout de «l'illusion d'optique » (p. 211), sinon du leurre. Loin de se résorber, les différences entre eux pourraient même se multiplier. Leur traque est ici menée par de nombreuses entrées: origine sociale, parcours de formation, revenus, valeur accordée au métier (prestige) et conditions d'exercice, travail prescrit et réel, sociabilités, ou encore pratiques culturelles. Finalement, sur l'ensemble de ces aspects, des clivages apparaissent. Si des différences anciennes, voire séculaires, demeurent entre les deux degrés, cet ouvrage montre très pertinemment comment elles se reconfigurent actuellement, changeant de visage du fait de multiples évolutions sociales. L'auteure n'en oublie pas moins les points communs qui lient aussi l'ensemble des enseignants aujourd'hui. D'un point de vue méthodologique, l'analyse s'appuie un dispositif solide : 1749 questionnaires et 60 entretiens semi-directifs réalisés auprès d'enseignants. Il s'agit du matériau de thèse de 
l'auteure, ici repris pour une nouvelle investigation. Le cadre théorique choisi est celui de la sociologie de la stratification sociale, qui pense les sociétés comme des compositions de couches sociales inégales et hiérarchisées.

2 Le chapitre 1 propose une analyse socio-historique de la manière dont l'institution scolaire appréhende les enseignants des premier et second degrés. L'étude de la formation, des hiérarchies et du travail prescrit, montre que, contrairement à certaines idées reçues, les réformes institutionnelles récentes ne vont pas dans le sens d'une unification de l'ensemble des enseignants, bien au contraire. Le chapitre 2 prend pour objet d'étude les inégalités de salaires, le travail réel, mais aussi les hiérarchies de statuts, notamment liées à des parcours universitaires hétérogènes (clivage dans les types d'études), entre les deux degrés. Sont également analysés des bougés récents sur la question du statut des enseignants par rapport à d'autres catégories d'actifs. On notera par exemple que les professeurs des écoles se sont rapprochés des cadres en termes de niveaux d'études et de rémunération. Dans le chapitre 3, on voit que les parents des enseignants des deux degrés sont aujourd'hui plus diplômés que jadis. Si les origines sociales des enseignants se sont élevées récemment, les enseignants du premier degré restent d'origine sociale plus modeste que ceux du second degré. Le chapitre 4 s'interroge sur la valeur donnée aux métiers de l'enseignement. L'impression d'exercer une profession peu valorisée apparaît chez tous les enseignants. Des distinctions se font cependant jour sur la valeur « intellectuelle» ou non du métier, bien plus présente du côté $d u$ second degré. Ceci peut être particulièrement frustrant pour les enseignants du premier degré d'aujourd'hui, qui ont réalisé un parcours d'étude bien plus conséquent que jadis. Le chapitre 5 se penche sur les conditions d'exercice du métier. Le travail enseignant s'avère aujourd'hui plus complexe, plus intense, mais également plus lourd au niveau de la charge de travail. L'exigence de polyvalence se développe en outre dans tous les degrés. Les jeunes enseignants exercent souvent dans des conditions délicates (publics difficiles, éloignement géographique du domicile). Ce chapitre se penche aussi sur les contrastes dans le rapport aux associations professionnelles, ainsi qu'à l'égard des perspectives de mobilité. Le chapitre 6 montre un désir général de se distancier d'une sociabilité liée à l'entre-soi enseignant, même si les sociabilités réelles demeurent de facto fortement liées au monde professionnel. Le chapitre 7 étudie les pratiques culturelles des enseignants. Si la consommation culturelle légitime s'avère affaiblie dans les premier et second degrés, la notion de culture légitime n'en demeure pas moins déterminante. Selon les parcours sociaux, l'éclectisme culturel et la consommation de biens culturels moins légitimes s'avèrent plus ou moins assumés. Plus les enseignants ont une origine sociale élevée, plus ils se sentent libres vis-à-vis de la culture légitime. On notera enfin qu'entre chacune de ses thématiques, assez hétérogènes, l'ouvrage établit des liens subtils : origine sociale et sociabilité (p.149), déclassement et sociabilité (p.173), ou encore consommation culturelle et revenus (p. 190).

3 En définitive, nous sommes en présence d'un ouvrage de grande qualité, dont le mérite est de proposer un portrait très actualisé des enseignants du premier et du second degrés. Une administration méthodique et patiente de la preuve est en outre mise en œuvre. À ce titre, il convient de mentionner que l'auteure mobilise de nombreuses recherches très récentes, établissant un dialogue heureux entre son matériau et la recherche sociologique actuelle. D'un point de vue scientifique, est ainsi produite une mise à jour utile sur des questions classiques (embourgeoisement ou non des 
enseignants, caractéristiques socioprofessionnelles du conjoint, etc.). Au passage, se voient battues en brèche certaines idées reçues sur la condition enseignante (travail réel des enseignants, rapport aux syndicats, appartenances politiques etc.). L'ouvrage semble ainsi destiné aux spécialistes mais aussi aux formateurs, étudiants ou acteurs du système éducatif, qui seraient intéressés par ces questions. C'est, au demeurant, le propre des ouvrages parus dans cette collection «Éducation et société », dirigée par Agnès Van Zanten.

4 S'il fallait formuler une critique, elle se situerait peut-être au niveau du primat accordé à la différence premier / second degré, laissant parfois inexplorés d'autre clivages. Quelles différences entre un professeur exerçant dans des zones urbaines ou rurales? Quels clivages entre un enseignant du premier degré exerçant en maternelle ou en élémentaire? De même, quid du genre? Croisées, toutes ces questions pourraient donner lieu à de nouvelles enquêtes passionnantes, approfondissant les problématiques ici traitées. Mais l'auteure reconnaît ces limites dès l'introduction, liées en partie aux réponses du questionnaire (p. 30-31), et l'on peut donc être sûr qu'elle développera ces questions dans ses travaux à venir. Enfin, bien que ce ne soit pas l'objet de l'ouvrage, on aurait aimé que des hypothèses soient proposées sur les liens entre ces évolutions des mondes enseignants et la question des choix pédagogiques mis en œuvre dans les classes. En s'appuyant sur d'autres travaux, quelques rares passages entrouvrent très légèrement ces questionnements : liens entre pratiques pédagogiques et valorisation intellectuelle de la profession dans le second degré, liée à l'exercice d'une seule discipline d'enseignement (p. 110-111), ou encore rapports entre élévation générale de l'origine sociale des enseignants et choix pédagogiques (p.73). Les conditions enseignantes contemporaines, ici éclairées, pourraient-elles expliquer en partie les types d'autorité aujourd'hui mis en œuvre, le recours ou non aux pédagogies liées à l'éducation nouvelle, ou encore la mise en œuvre ou non d'une logique inclusive vis-àvis des élèves de milieu populaire, que l'école française peine à faire réussir?

\section{AUTEURS}

\section{GHISLAIN LEROY}

Université Paris-Sorbonne - ESPE de l'Académie de Paris ; Université Paris Descartes, CERLIS 